\title{
LOS INTERESES DE SUDÁFRICA COMO BRIC
}

\section{The interests of South Africa as BRIC}

Carla Maria Morasso $^{1}$

Respondiendo al acrónimo lanzado por Goldman Sachs en el 2001, Brasil, Rusia, India y China se reunieron por vez primera en junio de 2009 en Ekaterimburgo, Rusia, con el objeto de conformarse en una plataforma donde compartir visiones sobre las oportunidades y desafíos de la globalización. Desde ese momento, el gobierno sudafricano encabezado por Jacob Zuma comenzó una activa campaña para ser invitado, ya que consideraba que el continente africano debía estar representado y Sudáfrica era el portavoz más idóneo para ser reconocido por los BRICs.

Desde Sudáfrica se tenía la percepción de que quedar fuera del grupo significaba la exclusión de África de la toma de desiciones en las estrategias globales Sur-Sur y dejarla a merced de los intereses económicos de las potencias emergentes. En palabras del director de Comercio e Industria, Tshediso Matona, el departamento de Relaciones internacionales y Cooperación debía trabajar en función de la inclusión de Sudáfrica en los BRIC porque “debían estar en ese club”, porque "pertenecían a él”2.

La situación representaba para el presidente Zuma un desafío extra, puesto que debía demostrar que estaba a la altura de la capacidad diplomática que había caracterizado a su predecesor. El gobierno de Thabo Mbeki (1999-2009), a quien se lo conocía como el "Presidente de Relaciones Exteriores", había logrado el

\footnotetext{
${ }^{1}$ Docente Facultad de Ciencia Política y Relaciones Internacionales UNR. Programa de Estudios América Latina Africa - Facultad de Ciencia Política y Relaciones Internacionales - Universidad Nacional de Rosario Doctoranda de Relaciones Internacionales UNR. Subcoordinadora del Programa de Fortalecimiento Institucional y Gestión Fiscal Provincial del Ministerio de Economía y Finanzas de la Nacion - BID. E-mail: carlam1980@hotmail.com.

${ }^{2}$ Citado en Kornegay (2009).
} 


\section{Conjuntura Austral}

reconocimiento internacional de Sudáfrica como potencia regional y como un actor con proyección internacional (Elíades, 2011).

El aparato diplomático sudafricano se puso en marcha y a lo largo de 2010 se realizaron las gestiones y visitas de Estado necesarias para promover la candidatura sudafricana. En el mes de abril Zuma viajó a Brasil, en el marco de una cumbre IBSA, y mantuvo un encuentro bilateral con el presidente Luiz Ignacio “Lula” Da Silva. En junio viajó a India a reunirse con el primer ministro Mannoham Singh, y en agosto a Rusia y China, para encontrarse con el presidente Dmitry Medvedev y el Premier Wen Jiabao, respectivamente. Posteriormente, en noviembre de 2010, Sudáfrica solicitó el ingreso al grupo en el marco de la cumbre del G-20 en Seúl. En esa ocasión, el presidente ruso, Dmitri Medvédev, declaró que el grupo valoraba positivamente la solicitud de ingreso sudafricana.

Las giras presidenciales y los esfuerzos de la cancillería tuvieron su rédito y finalmente Sudáfrica recibió la invitación esperada. En diciembre de 2010, la ministra de Relaciones Internacionales y Cooperación, Maite Nkoana-Mashabane, recibió un llamado telefónico de su par chino, Yang Jiechi, invitándo al país a participar de la tercera reunión del bloque ${ }^{3}$, que se realizaría en Sanya, China, en abril de 2011. Como organizador de la próxima cumbre, es comprensible que China haya anticipado la invitación. No obstante, algunos analistas señalan que la invitación de Sudáfrica a los BRIC por sobre la de otros candidatos, como México, Turquía o Indonesia, la promovió China por los intereses económicos que tiene en el África.

El ingreso lo confirmó el ministro de Finanzas indio, Pranab Mukherjee, en la reunión de los ministros de Finanzas del G20 en Paris, en febrero de 2011: "Hoy Sudáfrica ha sido reclutada formalmente para formar parte del grupo de países emergentes BRIC. A partir de ahora pasará a llamarse BRICS y este será un foro global

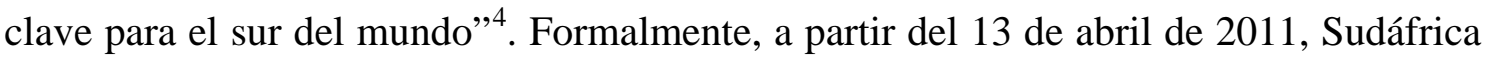

\footnotetext{
${ }^{3}$ La segunda reunión de jefes de Estado fue realizada en Brasilia, Brasil, en abril de 2010, y la cuarta reunión en Nueva Delhi, India, en marzo de 2012.

4 “Sudáfrica se une oficialmente al grupo económico BRIC”. Disponible en: http://actualidad.rt.com/economia/view/23814-Sud\%C3\%A1frica-se-une-oficialmente-al-grupoecon\%C3\%B3mico-BRIC
} 


\section{Conjuntura Austral}

participa del Foro BRICS con el objetivo de fortalecer su proyección internacional en base a la representación de los intereses del continente africano. A continuación, se presenta el lugar que ocupa el Foro en la política exterior sudafricana, dando cuenta de los intereses nacionales y de los desafíos y posibilidades que esta iniciativa presenta para Sudáfrica.

\section{El ingreso a los BRICs como líder regional}

Tras la admisión de Sudáfrica en el grupo las críticas no tardaron en llegar, sobre todo por las asimetrías latentes entre Sudáfrica y el resto de los BRIC. La economía sudafricana es considerablemente menor que la de sus pares, con un crecimiento que ha sido afectado por la crisis financiera internacional en los últimos años y una dinámica que no la coloca entre los próximos "mercados emergentes".

Los creadores del acrónimo no sitúan al país entre las próximas once economías emergentes, denominadas “Next 11”: México, Turquía, Irán, Corea, Indonesia, Pakistán, Filipinas, Egipto, Nigeria, Blangladesh y Vietnam. En este sentido, Jim O'Neill criticó el ingreso de Sudáfrica al grupo diciendo que esto "carecía de sentido”, que "Sudáfrica no pertenecía a los BRIC”, y que en todo caso, si se requería que el contimemte estuviera representado, Nigeria tenía una mejor performance para hacerlo ${ }^{5}$. Otros analistas financieros también consideraron que era un error esta incorporación. Desde el BBVA Research no se incluyó a Sudáfrica en el grupo de los "EAGLES” (economías emergentes que lideran el crecimiento global, por sus siglas en inglés), que son aquellos mercados emergentes cuya contribución al crecimiento mundial superará el promedio de las economías del G6 (es decir, el G7 excluido EE.UU.) en los próximos diez años ${ }^{6}$, y se señaló que el lugar junto a los BRIC debería haberlo tomado Egipto o Indonesia (García Herrero et. al., 2011).

No obstante las razones económico-financieras que aluden los críticos, el ingreso de Sudáfrica estuvo relacionado con factores geopolíticos que la convirtieron en un

\footnotetext{
${ }^{5}$ Hervieu, S., 2011, "South Africa gains entry to Bric club”, in The Guardian, 19 April. Disponible en: http://www.guardian.co.uk/world/2011/apr/19/south-africa-joins-bric-club

${ }^{6}$ China, India, Brasil, Indonesia, Corea del Sur, Rusia, México, Egipto, Taiwán y Turquía.
} 


\section{Conjuntura Austral}

socio deseable para los BRIC. El concepto inicial del acrónimo creado por O’Neill en base a proyecciones económicas se ha rebalsado en la práctica con la introducción de concepciones políticas. Las declaraciones finales de las reuniones de alto nivel de los BRICS reflejan la visión que comparten del mundo y las propuestas que impulsan para influir en los temas globales diferentes a los económicos, tales como la salud o el cambio climático. La incorporación de Sudáfrica reforzó la superación del BRIC financiero. Sudáfrica fue invitada por dos motivos princiaples: por su lugar en el continente africano, que la ubica como "puerta de enlace" al continente y portavoz de la región; y por su gran mercado de capitales, que es una excepción en África, y su economía preponderante.

Algunos datos dan cuenta del lugar de Sudáfrica en el continente: alberga el 6\% de la población de África, es la mayor economía del continente y genera el 25\% del PBI del mismo, cuenta con el $45 \%$ de la producción minera africana y representa el 50\% del poder adquisitivo ${ }^{7}$. Además, el país brinda estabilidad institucional, un mercado financiero integrado internacionalmente, que está entre los 20 mayores del mundo, y un manejo macroeconómico que ubica al país entre los 20 mejores destinos para realizar inversiones en el mundo (Banco Mundial, 2011).

La superioridad económica sudricana es uno de los motivos por lo cual el país es visto por los actores extra regionales como un líder en el continente, como el negociador nato portador de la agenda africana, independientemente de las críticas que pueden manifestar sus vecinos a este rol.

También en la autopercepción de las elites gobernantes sudafricanas el país es un lider regional casi natual. De acuerdo con Gladys Lechini, esta idea se encuentra presente en los distintos modos de inserción internacional: "En toda la historia de Sudáfrica, se puede observar en forma implícita o explícita, tanto en los discursos como en las acciones de política exterior, a un país que se percibe como líder o potencia regional africana. Los propósitos subyacentes a la promoción de este aspecto del interés nacional sudafricano, están vinculados a posibilitar la permanencia, consolidación y

\footnotetext{
7 Datos proporcionados por la Embajada de Sudáfrica en Argentina. Disponible en: http://www.embajadasudafrica.org.ar/espa/comercio/home/home.php
} 


\section{Conjuntura Austral}

crecimiento de los intereses económicos y financieros de las inversiones en el país y en el cono sur de África, sean de sudafricanos o de extranjeros” (Lechini, 2010: 202).

Desde la instauración del nuevo modelo democrático y multirracial en 1994, la reinserción internacional fue uno de los objetivos alcanzados del Congreso Nacional Africano (ANC). Para ello, apostó a afianzar su lugar de liderazgo en África junto con el desarrollo de vínculos con los países desarrollados y los emergentes en desarrollo. Una constante en la política exterior sudafricana es la activa participación en todas las cuestiones importantes de África, mediando en los conflictos regionales, actuando en todas las instancias de cooperación e integración económica y jugando el rol de intermediario entre los países más pobres de la región y los desarrollados.

La idea de ser un "buen vecino” que planteaba Mandela, aludiendo al propósito de ayudar a resolver los problemas continentales que habían sido exacerbados por los gobiernos del apartheid, fue seguida por el "Renacimiento Africano". Bajo el gobierno Mbeki se impulsaron las instancias cooperativas y los mecanismos para impulsar el desarrollo sustentable conjunto, principalmente a través de la SADC, la Unión Africana (UA) y la Nueva Asociación Económica para el Desarrollo Africano (NEPAD) ${ }^{8}$.

Este activismo en el continente es visto desde dos veredas opuestas. Por una parte, están quienes consideran que Sudáfrica desarrolla una política cooperativa, donde no procura solamente su crecimiento de manera aislada porque el mismo está estrechamente ligado con el desarrollo de África. Enfrente se encuentran los que consideran que Sudáfrica actúa como hegemón en busca de sus propios intereses, para lo cual se apoya en su superioridad militar y económica. Desde aquí, las instituciones regionales promovidas por el gobierno sudafricano son vistas solamente como un medio para plasmar los intereses sudafricanos y se señala que las empresas sudafricanas sólo prentenden maximizar sus ganancias invadiendo los mercados africanos con agresivas estrategias de mercadeo. Por ello, algunos analistas, como Chevallier, señalan que "a

\footnotetext{
${ }^{8}$ Esta es una iniciativa anclada en la UA e impulsada por Mbeki con la idea de que sea una herramienta para lograr un continente con menos violencia, corrupción y pobreza, pero también para fortalecer el liderazgo sudafricano al constituir al país en un interlocutor reconocido mundialmente. Actualmente, la NEPAD es reconocida mundialmente y tiene el apoyo de Naciones Unidas, el G-8 y la UE en su accionar continental.
} 


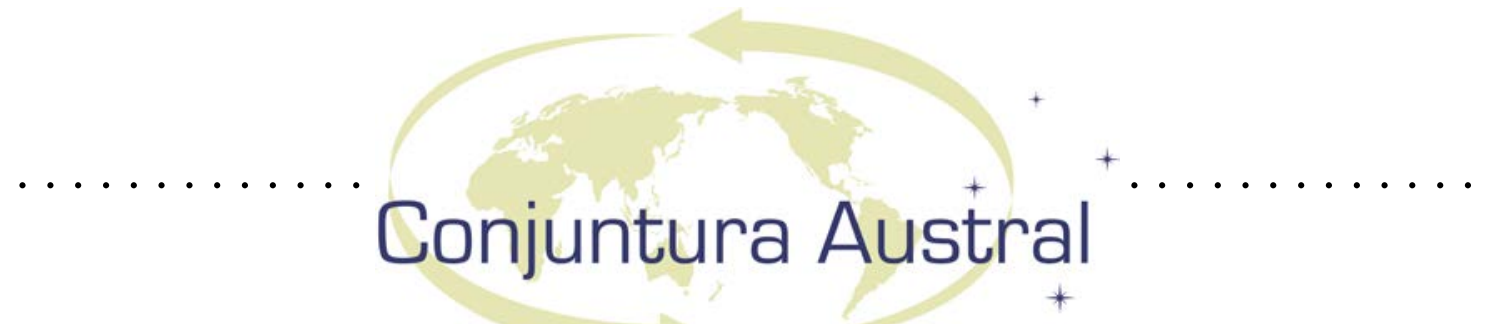

pesar de su ambicioso programa para África, Sudáfrica no tiene verdaderos aliados de peso en el continente, y pocos amigos comparten valores comunes” (Chevallier, 2008: 169).

Ante esta situación, la política exterior sudafricana se presenta como “ambivalente”. Por momentos el país actúa como un líder regional, pero en otras circunstancias evita realizar acciones que podrían llevar a sus vecinos a percibirlo como un hegemón, tomando desiciones que considera tendrán mejor aceptación en la región y disiparán los temores que causa la hegemonía. Esta ambivalencia lleva a que despliegue entonces una diplomacia excesivamente cautelosa con el continente y que se esté siempre sensible a las acusaciones de dominación (Kornegay, 2010), desarrollando en casos controvertidos lo que se ha denominado “diplomacia silenciosa”.

En cuanto a los BRIC, queda claro que no obstante las diferentes percepciones sobre la hegemonía sudafricana en el continente, fue clave el rol de Sudáfrica como líder de la región para que sea invitado a participar del foro. Los BRIC están interesados en los recursos de poder blando -status democrático, modelo económico, influencia cultural e ideológica-, a través de los cuales Sudáfrica promueve la estabilidad regional y el acceso a mercados y materias primas y suma la voluntad de sus pares en iniciativas conjuntas.

\section{El foro BRICS en la política exterior}

Desde el año 2005, los ejes estratégicos de la política exterior sudafricana plasmados en los planes estratégicos trianuales del Departamento de Relaciones Internacionales y Cooperación (DIRCO) ${ }^{10}$, se mantienen casi intactos: el continente africano; las relaciones Sur-Sur; y el sistema de gobernanza global. De acuerdo con Wheeler (2011), la política exterior de Zuma puede describirse como de “cuidado y

\footnotetext{
${ }^{9}$ El ejemplo más claro es la política sudafricana hacia el gobierno de Mugabe. Frente a la crisis en Zimbawe, Mbeki desarrolló una estrategia basada en la negociación entre los actores, sin declaraciones públicas a favor de los derechos humanos y el Estado de derecho y evitando aludir a sanciones y posibles intervenciones en el país, señalando que con ello sólo se agravaría la situación interna de un país cuyos problemas no eran una amenaza para la seguridad internacional.

${ }^{10}$ Hasta 2010 mantuvo el nombre de Departamento de Relaciones Exteriores. El cambio en el nombre fue para dar cuenta de una visión más cooperativa de la política exterior con los vecinos y más relacionada con la política interna (Gruzd, 2009).
} 


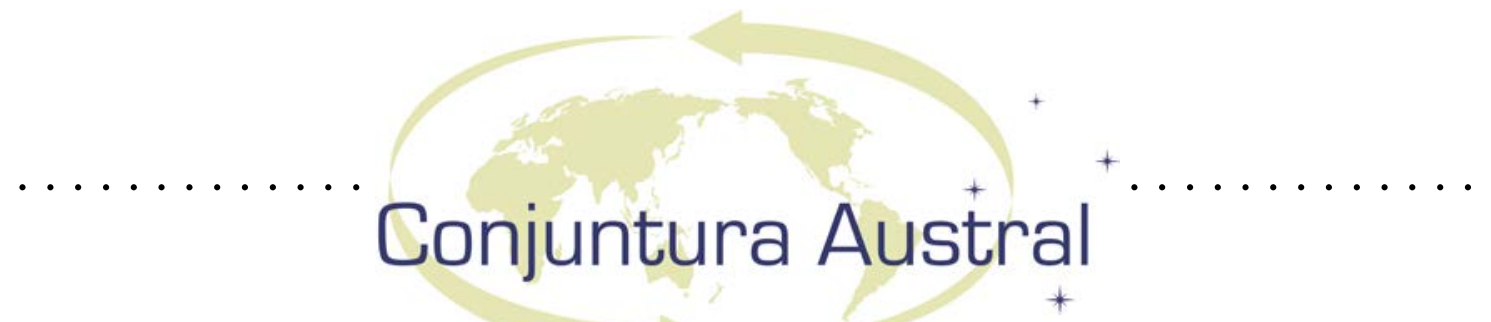

mantenimiento" de lo que fue creado por Mbeki y en este sentido se conservan los objetivos principales.

El “Plan Estratégico 2011-2014” señala las siguientes prioridades de la política exterior sudafricana:

1- La agenda africana y el desarrollo sustentable: profundizar la contribución con la seguridad y la estabilidad regional y continental y el desarrollo; fortalecer las relaciones bilaterales; fortalecer la presencia en las misiones de paz y reconstrucción post-conflicto del Consejo de Seguridad de Naciones Unidas (CSNU) ${ }^{11}$ y del Consejo de Seguridad y Paz de la Unión Africana (AUPSC) ${ }^{12}$.

2- Integración política y económica de la Comunidad de Desarrollo de África Austral (SADC).

3- Fortalecimiento de las Relaciones Sur-Sur: compromiso con el Movimiento de los Países No Alineados y el G77 + China; implementación de los acuerdos alcanzados en el marco de IBSA; revitalización de la Nueva Asociación Estratégica Africana-Asiática (NAASP).

4- Fortalecimiento de los vínculos con formaciones estratégicas del Norte: el objetivo final es avanzar en el desarrollo de políticas para solucionar problemáticas africanas y del Sur en general. La participación en el G-20, la OMC, el G-8 y la OCDE apuntan en esta dirección.

5- Participar del sistema de governanza global: contribuir a través del sistema multilateral a la estabilidad socio-económica global y regional.

6- Fortalecimiento de las relaciones económicas y políticas: con América Latina y el Caribe se subrayan los vínculos con Brasil, la Cumbre África - Sudamérica (ASA) y la ratificación del acuerdo de preferencias comerciales MERCOSUR-SACU ${ }^{13}$.

\footnotetext{
${ }^{11}$ Durante el período 2011-2012, Sudáfrica tiene una silla de miembro no permanente en la CSNU.

${ }^{12}$ Sudáfrica está involucrada en el trabajo de reconstrucción de la paz en la República Democrática del Congo, en Sudán y en las Comores. Bajo los mandatos de la SADC y la AU, facilita la mediación y los esfuerzos de reconstrucción de la paz en Sudán, Zimbawe, Madagascar, Lesotho y la Región de los Grandes Lagos.

${ }^{13}$ El acuerdo fue firmado en abril de 2009 y Sudáfrica lo ratificó en junio de 2010, mientras que no fue ratificado aún por los miembros del MERCOSUR.
} 


\section{Conjuntura Austral}

En este marco, BRICS significa para Sudáfrica un lugar especial desde donde incidir en el sistema de governanza global en función de los intereses que mantiene en África. La concepción básica a partir de la cual participa en el Foro está expresada en el documento denominado "White Pape”, el cual fue elaborado por el DIRCO y elevado al parlamento.

En este punto, cabe señalar que para muchos especialistas sudafricanos el gobierno de Zuma ha optado por un proceso de elaboración de la política exterior más inclusivo, donde se consulta a los empresarios, académicos, sindicalistas y representantes de la sociedad civil. Esta apertura diferencia a esta adminstración de la de Mbeki, donde el diseño estaba concentrado en la presidencia (Wheeler, 2011).

En el "White Paper” se define a Sudáfrica como un país multifacético, multicultural y multiracial que lleva adelante una diplomacia basada en la filosofía del Ubuntu, que significa "humanidad”, y refleja la idea de que nuestra propia humanidad se afirma cuando confirmamos la humanidad de los otros. Esta filosofía se encuentra en la conciencia nacional sudafricana y ha tenido un papel principal en el proceso de democratización. De acuerdo con la DIRCO, considerar los temas globales desde este lugar hace que Sudáfrica tenga una singular visión de lo que debe ser un mejor mundo y una diplomacia con rasgos propios.

Este documento también explicita que los principios de la política exterior reflejan el interés nacional al reconocer que los estados son interdependientes y promover la cooperación sobre la confrontación y la competición, motivo por el cual se procura genera asociaciones a través del mundo. Las mismas descansan en el espíritu del internacionalismo, el panafricanismo y la solidaridad Sur-Sur, rechazando todo tipo de colonialismo y promoviendo una estructura global más equitativa y democrática.

Por otra parte, el “White Paper” plasma la visión de Sudáfrica para el año 2025 como un miembro influyente de la comunidad internacional con una economía competitiva que sustente el desarrollo nacional y regional, avanzando en la lucha contra la pobreza, el desempleo y la desigualdad. Señala que hay tendencias sin precedentes en el sistema internacional que les brindan a los países la oportunidad de maximizar su influencia al liderar discusiones en temas específicos y en sus regiones a través de 


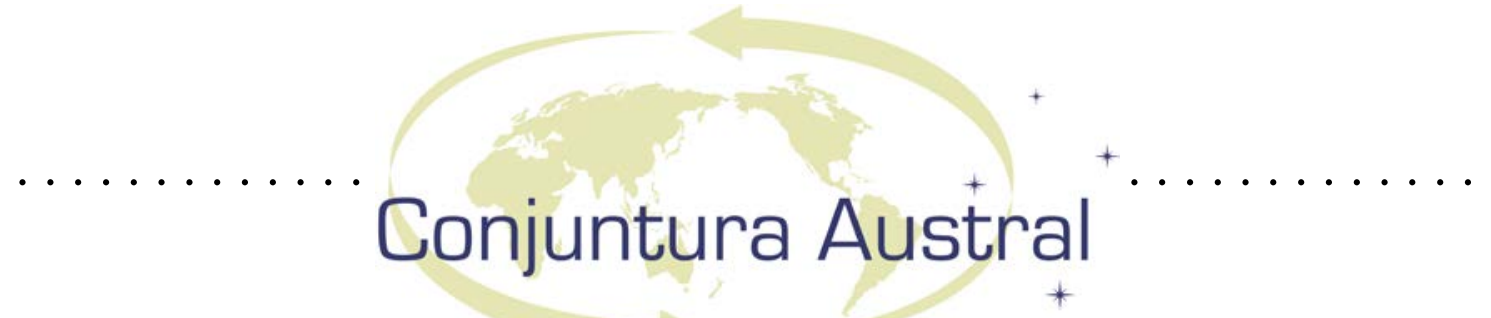

instituciones, iniciativa política y soluciones originales. En este sentido, reconoce que Sudáfrica ha aumentado su influencia internacional a partir del rol de liderazgo en el continente africano y que el próximo desafío estratégico es tomar la iniciativa en la definición de un nuevo orden mundial.

Asimismo, es interesante rescatar del documento oficial la posición sudafricana ante un posible impacto negativo en la solidaridad Sur-Sur debido a la existencia de ciertos países en desarrollo, que por sus capacidades y nivel de integración en las estructuras globales, ya están diferenciados y buscan diferenciarse a sí mismos del resto del mundo en desarrollo. Sobre esto, se subraya que el lugar de Sudáfrica es mantenerse como un puente entre los poderosos y los menos desarrollados y llevar adelante la agenda del mundo en desarrollo.

En este marco, se observa que la participación en el foro BRICS es un paso táctico más en la consecución del objetivo de máxima: participar con poder de desición en delinear nuevos parámetros internaciones. La desición del gobierno de Zuma de movilizar sus recursos para ingresar al grupo no fue aislada, sino que formó parte de la estrategia diplomática iniciada por Mbeki de conformar alianzas Sur-Sur interregionales para incrementar los márgenes de maniobra internacional en post de intereses africanos. También puede desprenderse de lo expuesto en el "White Paper", que siendo los BRIC países en desarrollo con caracterísiticas de potencias emergentes, que se “diferencian” de sus pares claramente, Sudáfrica buscará en el interior del grupo mantener los principios de solidaridad horizontal y poner sobre la mesa las problemáticas panafricanas.

Por último, cabe señalar que el activismo internacional sudafricano es uno de los atributos que le valieron su ingreso a los BRICS en 2011. A diferencia de México, Egipto o Indonesia, Sudáfrica se ha involucrado en temáticas globales y comparte con los BRIC la idea de reformular las estructuras de governanza global para adaptarlas a las nueva realidad multipolar.

La participación en el G20 como único país africano, la conformación de IBSA, su selección para ser miembro no permanente del CSNU en dos períodos próximos (2007-2008 y 2011-2012), la elección de la ex ministra de Relaciones Internacionales y 


\section{Conjuntura Austral}

actual ministra del interior, Nkosazana Dlamini-Zuma, como presidenta de la Comisión de la Unión Africana (UA) ${ }^{14}$, el rol de mediador del presidente en conflictos africanos, la organización de la XVII Conferencia de Cambio Climático ${ }^{15}$ y de la I Cumbre Global de la Diáspora Africana ${ }^{16}$, son algunos de los ejemplos que muestran la reciente presencia internacional del país.

\section{A modo de cierre: las expectativas sudafricanas}

El ingreso sudafricano al club de los BRICS encuentra sus principales causas en la figura de liderazgo que se le atribuye a Sudáfrica en el continente, a su diplomacia activa y a la cosmovisión del sistema internacional que comparte con los miembros originarios del grupo.

En cuanto a los beneficios y oportunidades que Sudáfrica encuentra en haberse convertido en un BRICS, hay variados aspectos a tener en cuenta. En primer lugar, la oportunidad de tener voz y voto en el proceso de toma de desición de las cuestiones globales junto a los poderes emergentes que procuran reestructurar el orden internacional. En palabras del presidente Zuma “(...) Ahora somos co-arquitectos de un nuevo sistema internacional equitativo. Este nuevo orden beneficiará a toda la humanidad y se propone asegurar una prosperidad compartida para todos. Lo que distigue a cada uno de los países BRICS es el valor y la importancia que le otorgamos al desarrollo. Compartimos el compromiso de asegurar que nuestros pueblos se beneficien al mayor nivel posible del crecimiento global y que los beneficios de la expansión económica se compartan equitativamente" ${ }^{17}$.

No obstante, desde un lugar crítico, algunos se preguntan hasta qué punto ser miembro de un club exclusivo, que pretende tener los privilegios que disfrutan los países del Norte, no alejará a Sudáfrica del resto de los países en desarrollo, a pesar de que el gobierno intente lo contrario (Qobo, 2010).

\footnotetext{
${ }^{14}$ La elecciónt tuvo lugar en la XIX Cumbre del bloque en Etiopía en julio de 2012.

${ }^{15}$ Realizada en Durban entre el 28 de noviembre y el 11 de diciembre de 2011.

${ }^{16}$ Realizada en Sandton el 25 de mayo de 2012.

17 Address by President Jacob Zuma to the plenary of the third BRICS leaders meeting, "Broad vision, shared prosperity”, Sanya, Hainan Island, People's Republic of China, 14 Apr 2011.
} 


\section{Conjuntura Austral}

En segundo lugar, Sudáfrica tiene la oportunidad de ser la voz del continente, de representar los intereses africanos. En palabras de la ministra Nkoana-Mashabane, “(...) como miembros de BRICS articiparemos activamente en todas sus actividades. En nuestro esfuerzo por expandir la Agenda Africa y asegurar el desarrollo socioeconómico sustentable continuaremos impulsando las posiciones sudafricanas vis-à-vis las prioridades NEPAD/SADC via BRICS e IBSA" ${ }^{18}$.

Como señalan Alden y Sidiropoulos (2011), en medio de una transformación de la economía política global que se disputa fuera del continente, se profundiza la competencia en África entre los actores del Norte y el Sur, e incluso dentro de cada grupo, siendo possible que el resultado sea a expensas de los intereses africanos. Por ello, es crucial la existencia de un liderazgo africano responsable que medie y maneje estas fuerzas en beneficio del desarrollo regional.

Es por tal motivo que la interlocución sudafricana no se pretende sólo ante el Norte, sino también ante aquellos países en desarrollo emergentes que están explayando sus intereses económicos y geoestratégicos en África. El llamado "nuevo reparto” del continente entre Brasil, India, China y Rusia es un tema que preocupa a diversos analistas y gobernantes, ya que paulatinamente las inversiones, el comercio y la cooperación técnica de estos paises desplazan a las potencias coloniales que históricamente tuvieron presencia en África ${ }^{19}$.

De acuerdo con Kornegay (2010), a medida que la cooperación Sur-Sur le confiere mayor importancia a la diplomacia económica y al posicionamiento geoeconómico, la competencia entre los emergentes se hace evidente y deja a Sudáfrica inserta en medio de competidores, con los cuales también colabora, en su propia zona de influencia. Es así que muchas veces Sudáfrica se presenta como el punto de contacto para las potencias externas en su ingreso a África. Es el caso de China, que muchas veces toma a Sudáfrica como un “co-conspirador”, tal el ejemplo de la vinculación del Standard Bank con el Banco Comercial de China.

\footnotetext{
18 South Africa Government, Strategic Plan 2011-2014, “Mensaje de la ministra de Relaciones Internacionales y Cooperación”, página 4.

${ }^{19}$ Ver Lechini (2012), UNECA (2011); UNCTAD (2010); Schoeman (2011).
} 


\section{Conjuntura Austral}

Por otra parte, no debe perderse de vista las dificultades que implica obtener consensos en un continente con grandes heterogeneidades regionales y países que hasta disputan el lugar de África en sus sub-regiones, como el caso de Nigeria en el Oeste. Las limitaciones que debe enfrentar Pretoria para la creación de una diplomacia panafricana coherente y convincente son numerosas y la cuestión de los recursos escasos, tanto humanos como económicos, no es un límite menor.

Por último, está en discusión si ser parte de los BRICS traerá grandes beneficios económicos y comerciales para el país. Por un lado, coincidiendo con las declaraciones gubernamentales, se señala que es una oportunidad para que Sudáfrica se promocione a sí misma como un destino para las inversiones y pueda lograr reducir las barreras comerciales con sus socios. Una reunión anual con cuatro de las economías más dinámicas del mundo pueden crear un espacio de alto nivel para promover la actividad comercial (Alden y Sidiropoulos, 2011). Por otra parte, se subraya que Sudáfrica no necesita ser parte de los BRICS para maximizar la cooperación económica con estos países, ya que esto puede hacerlos bilateralmente. Además, se cuestionan las barreras arancelarias y para-arancelarias, que los cuatro mercados han profundizado en el marco de la crisis financiera internacional, tanto como la competencia con Sudáfrica en diversos sectores, como el del acero, el automotriz y el textil (Soko, 2010).

La ambiciosa política exterior sudafricana ha considerado un éxito el ingreso a los BRICS. De ahora en más dependerá de cuán efectiva es para determinar objetivos prioritarios, acordes tanto al interés nacional como al pan-africanismo que pretende representar, y para desarrollar estrategias dentro de un grupo que no tiene la cohesion de IBSA y cuyas relaciones han sido caracterizadas como simultáneamente cooperativas y competitivas, particularmente en África. 


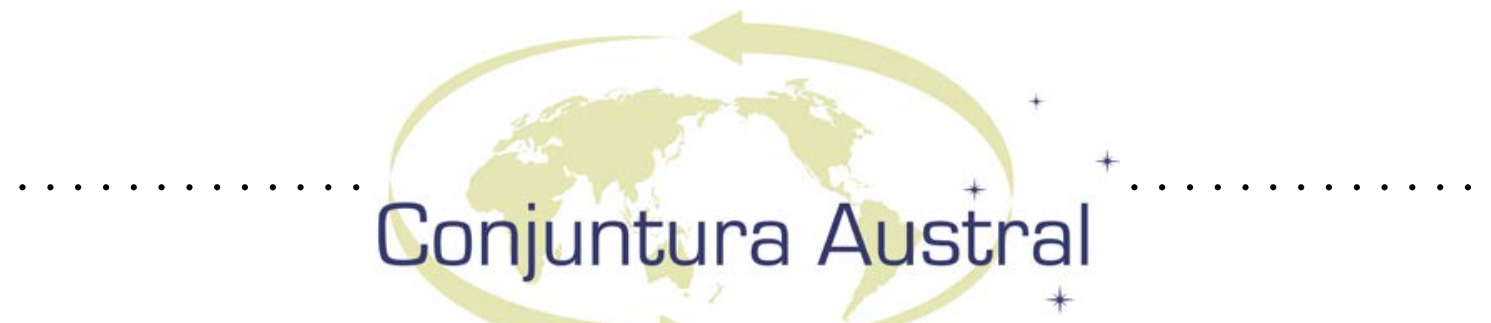

\section{REFERÊNCIAS}

ALDEN C. and SIDIROPOULOS, E., 2011, South Africa, Africa and the BRICS: The apex of the South?, SAIIA. Disponible en: http://www.saiia.org.za/feature/southafrica-africa-and-the-brics-the-apex-of-the-south.html

CHEVALLIER, R., 2008, “Thabo Mbeki y la agenda exterior de Sudáfrica”, en Política Exterior, $N^{\circ}$ 123, mayo/junio.

ELÍADES, E., 2011, “El papel asertivo de Sudáfrica como potencia regional”, presentación en el XII Simposio Electrónico Internacional África Una Mirada al Siglo XXI, organizado por CEID / Univesidad de Externado.

GARCÍA HERRERO, A.; NAVIA, D., NIGRINIS, M., 2011, Debería ser Sudáfrica un BRIC?, BBVA Reserach, Observatorio Económico, Hong Kong, enero. Disponible en: http://www.asia.udp.cl/Informes/2011/sudafrica_bric.pdf

GROBBELAAR, N., CHEVALLIER, R. and PORTER-ERASMUS, C., 2011, South Africa's ambitious foreign policy. Disponible en:

http://www.saiia.org.za/feature/south-africa-s-ambitious-foreign-policy.html

GRUZD, S., 2009, Foreign Policy under Zuma: Change of Style or Substance?.

Disponible en: http://www.saiia.org.za/governance-and-aprm-opinion/foreign-policyunder-zuma-change-of-style-or-substance.html

HERVIEU, S., 2011, “South Africa gains entry to Bric club”, in The Guardian, 19 April. Disponible en: http://www.guardian.co.uk/world/2011/apr/19/south-africa-joinsbric-club

KORNEGAY, F., 2009, “South Africa excluded as an emerging economic power?”, in The Sunday Independent, 29 June. Disponible en:

http://www.newsfromafrica.org/newsfromafrica/articles/art_11630.html

KORNEGAY, F., 2010, “A África do Sul como potência emergente: dilemas especiais e dimensões de segurança”, en Fagundes Visentini, Paulo y Danilevicz Pereira, Analúcia (org.) África do Sul: História, Estado e Sociedade, FUNAG/CESUL, Brasília.

KORNEGAY, F., 2012, BRICS in search of identity. Disponible en: http://igd.org.za/home/179-brics-in-search-of-identity

LECHINI, G., 2010, “Sudáfrica. La identidad nacional como construcción y el liderazgo regional como destino manifiesto”, en BUSSO, A. (comp.) Fuerzas profundas e 


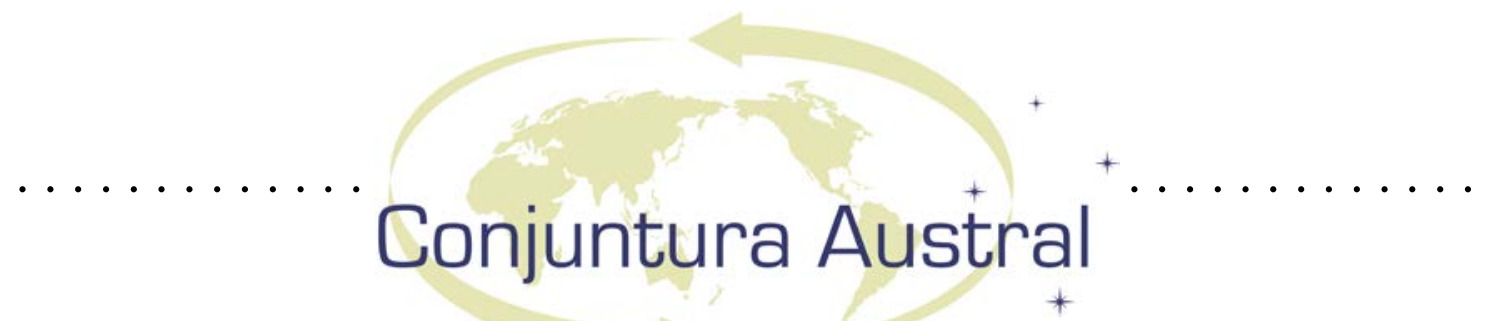

identidad. Reflexiones sobre su impacto en la política exterior: un recorrido de casos, UNR Editora, Rosario.

LECHINI, G., 2012, “BRICS e Africa: A grande incógnita”, en Boletim de Economia e Política Internacional, IPEA, $N^{\circ}$ 9, enero-marzo, Brasilia.

NAIDOO, S., 2012, “South Africa's presence 'drags down Brics'”, in The Mail \& Guardian, 21 March. Disponible en: http://mg.co.za/article/2012-03-23-sa-presencedrags-down-brics/

NARDI, V., 2011, El ingreso de Sudáfrica abre las puertas de África a los BRICS, Programa de Estudios América Latina-África, PRECSUR, UNR, Rosario, Julio. Disponible en: http://www.catedrarrii.com.ar/docs/Sudafrica\%20BRICS.pdf

QOBO, M., 2010, “The BRIC Pitfalls and South Africa’s Place in the World”, in The Sunday Independent, 17 April. Disponible en: http://www.saiia.org.za/great-powersafrica-opinion/the-bric-pitfalls-and-south-africa-s-place-in-the-world.html

SCHOEMAN, M., 2011, “Of BRICs and Mortar: The Growing Relations between Africa and the Global South”, in The International Spectator: Italian Journal of International Affairs.

SOKO, M., 2010, "South Africa and the BRICs: A Crisis of Identity in Foreign Policy”, in The Mail \& Guardian, 7 January. Disponible en: http://www.saiia.org.za/greatpowers-africa-opinion/south-africa-and-the-brics-a-crisis-of-identity-in-foreignpolicy.html

WHEELER, T., 2011, South Africa's foreign policy objectives: Reviewing the White Paper. Disponible en: http://www.saiia.org.za/feature/south-africa-s-foreign-policyobjectives-reviewing-the-white-paper.html

Address by President Jacob Zuma to the plenary of the third BRICS leaders meeting, Broad vision, shared prosperity, Sanya, Hainan Island, People's Republic of China, 14 Apr 2011. Disponible en:

http://www.info.gov.za/speech/DynamicAction?pageid=461\&sid=17783\&tid=32020

Banco Mundial, 2011, South Africa Economic Update. Focus on savings, investments, and inclusive growth, Washington.

United Nations Economic Commission for Africa (UNECA), 2011, African Economic Outlook.

Speech by Minister Maite Nkoana-Mashabane to the South African Institute off International Affairs (SAIIA) on the topic The relationship between South Africa and 


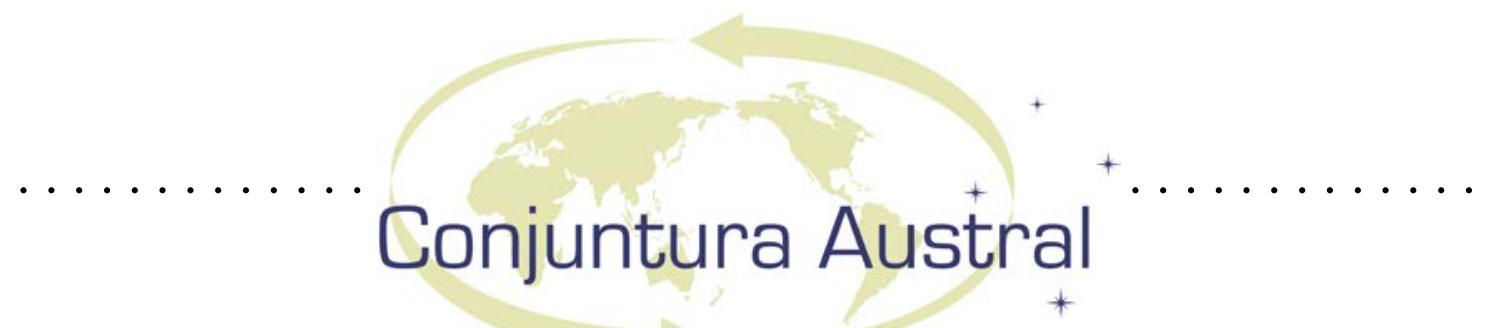

the Emerging Global Powers, 1 November 2010. Disponible en:

http://www.saiia.org.za/images/stories/saiia/saia_spe_min_maite_nkoana_mashabane_2 0101101.pdf

South Africa Government, Building a Better World: The Diplomacy of Ubuntu, White Paper, Final Daft, 13 May 2011. Disponible en:

http://www.info.gov.za/view/DownloadFileAction?id=149749

South Africa Government, Strategic Plan 2011-2014. Disponible en:

http://www.dfa.gov.za/department/strategicplan\%202011-2014/index.htm

South Africa Government, Strategic Plan 2010-2013. Disponible en:

http://www.dfa.gov.za/department/strategic\%20plan\%202010-

2013/strategic\%20plan\%202010-2013.pdf

United Nations Conference on Trade and Development (UNCTAD), 2010, Economic Development in Africa Report 2010. South-South Cooperation and the New Forms of Development Cooperation.

"S. Africa wants to be 5th "BRIC", Russia says", Reuteres África, 12 de noviembre. Disponible en: http://af.reuters.com/article/topNews/idAFJOE6AB06920101112

China invita a Sudáfrica a los BRIC, CNN, 24 de diciembre de 2010. Disponible en: http://www.cnnexpansion.com/economia/2010/12/24/china-invita-a-sudafrica-a-los-bric

Artigo recebido dia 02 de dezembro de 2012. Aprovado em 03 de março de 2013. 


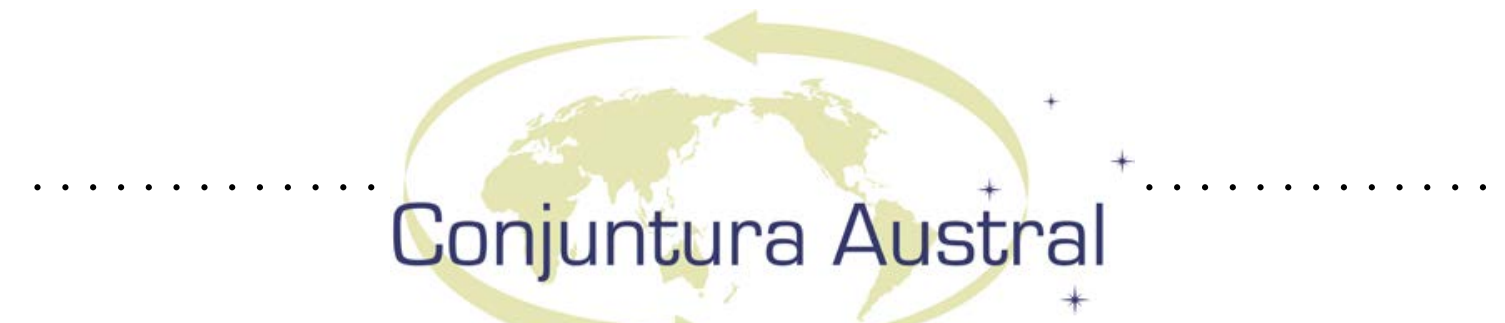

\section{RESUMO}

O objetivo do presente trabalho é examinar as iniciativas desenvolvidas pelo governo do África do Sul para formar parte dos BRICs (Brasil, Rússia, Índia e China) e identificar seus interesses no grupo. África do Sul pretende reforçar o seu papel de potência regional no continente africano e ao mesmo tempo quer ter o estatuto de país emergente. A política externa sul-africana encontra-se ante uma nova etapa de desafios provenientes da consolidação do seu rol de potência média e seu acionar no grupo pode contribuir a seus objetivos no atual cenário internacional.

\section{PALAVRAS-CHAVE}

África do Sul, BRICS, Política Externa.

\section{RESUMEN}

El objetivo de este trabajo es examinar las iniciativas desarrolladas por el Gobierno de Sudáfrica para ser parte de los BRIC (Brasil, Rusia, India y China) y identificar sus intereses en el grupo. Sudáfrica tiene la intención de reforzar su papel como potencia regional en África y al mismo tiempo quieren tener el estatus de un país emergente. La política exterior de Sudáfrica está frente a una nueva etapa de desafíos de la consolidación de su lista de potencia media y de su grupo de disparo puede contribuir a sus objetivos en el actual escenario internacional.

\section{PALABRAS CLAVE}

Sudáfrica, BRICS, Política Exterior.

\section{ABSTRACT}

The aim of this paper is to examine the initiatives developed by the government of South Africa to be part of the BRIC (Brazil, Russia, India and China) and identify their interests in the group. South Africa intends to strengthen its role as a regional power in Africa and, at the same time, it wants to have the status of an emerging country. The external policy of South Africa lies in a new phase of challenges from the consolidation of itself as an average power, and if it is added to the group, it can contribute to goals in the current international scenario.

\section{KEYWORDS}

South Africa, BRICS, External Policy. 